\title{
Fluorescent microparticles for sensing cell microenvironment oxygen levels within 3D scaffolds
}

\author{
Miguel A. Acosta, Patrick Ymele-Leki, Yordan V. Kostov ${ }^{\star}$, and Jennie B. Leach ${ }^{\star}$ \\ Department of Chemical and Biochemical Engineering, University of Maryland, Baltimore County \\ (UMBC), 1000 Hilltop Circle, ECS 314, Baltimore, MD 21250, USA
}

\begin{abstract}
We present the development and characterization of fluorescent oxygen-sensing microparticles designed for measuring oxygen concentration in microenvironments existing within standard cell culture and transparent three-dimensional (3D) cell scaffolds. The microparticle synthesis employs poly(dimethylsiloxane) to encapsulate silica gel particles bound with an oxygen-sensitive luminophore as well as a reference or normalization fluorophore that is insensitive to oxygen. We developed a rapid, automated and non-invasive sensor analysis method based on fluorescence microscopy to measure oxygen concentration in a hydrogel scaffold. We demonstrate that the microparticles are non-cytotoxic and that their response is comparable to that of a traditional dissolved oxygen meter. Microparticle size $(5-40 \mu \mathrm{m})$ was selected for microscale-mapping of oxygen concentration to allow measurements local to individual cells. Two methods of calibration were evaluated and revealed that the sensor system enables characterization of a range of hypoxic to hyperoxic conditions relevant to cell and tissue biology (i.e., $p \mathrm{O}_{2} 10-160 \mathrm{~mm} \mathrm{Hg}$ ). The calibration analysis also revealed that the microparticles have a high fraction of quenched luminophore $(0.90 \pm$ 0.02 ), indicating that the reported approach provides significant advantages for sensor performance. This study thus reports a versatile oxygen-sensing technology that enables future correlations of local oxygen concentration with individual cell response in cultured engineered tissues.
\end{abstract}

\section{Keywords}

Tissue engineering; Hypoxia; Optical sensors; Microspheres; Poly(dimethylsiloxane)

\section{Introduction}

Understanding the mechanisms underlying how cells respond to cues from their microenvironment will ultimately lead to improved methods to control cell behavior in tissue replacement therapies. Oxygen concentration is one such cue that directly impacts cell response; a notable example of this effect is the growth of new capillaries into tissue regions that have low oxygen content. Oxygen is required for the aerobic metabolism of carbon compounds, and as such, it is one of the critical parameters (along with $\mathrm{pH}$, temperature, and nutrient supply) that impacts cell viability. Furthermore, hypoxia has been linked with the progression of diseases such as cancer, where it plays a role in tumor resistance to radiation treatment [1-3], and hyperoxia can cause cell death via the formation of reactive oxygen species $[4,5]$.

\footnotetext{
* Corresponding authors. Tel.: +1 410455 8152; fax: +1 410455 1049. jleach@umbc.edu (J.B. Leach).

Appendix. Supplemental data: Supplementary data associated with this article can be found, in the online version, at doi:10.1016/ j.biomaterials.2009.02.021.
} 
It is well known that oxygen supply becomes a limiting factor during the culture of highly metabolic tissues and tissue masses with thicknesses greater than $100 \mu \mathrm{m}$. This is mainly a result of the lack of vascularization in tissues cultured in vitro and the low solubility of oxygen in the culture medium, as an oxygen molecule only diffuses $\sim 100 \mu \mathrm{m}$ away from the surface of a tissue construct before it is consumed [6]. As a consequence of these limitations, cells at the surface of large tissue constructs are usually viable, whereas cells at the center of the construct are hypoxic and necrotic.

A number of studies have attempted to correlate oxygen concentration to the success of engineered tissues. For example, Radisic et al. demonstrated that oxygen diffusion is a limiting factor during the culture of $1.5 \mathrm{~mm}$-thick cardiac tissue constructs [7]. In their study, levels of $\alpha$-actin, troponin- 1 , and connexin- 43 , which are related to the contractile behavior of cardiac myocytes and are associated with physiological behavior, were reported to decrease at the center of the tissue construct, where the cells were experiencing hypoxia. In addition to cardiac tissue engineering, oxygen concentration has also been explored as a means to modulate chondrogenesis [8] and direct stem cell differentiation [9].

Thus, it is evident that oxygen plays an important role in cellular function and that understanding of the mechanisms underlying cell response to changes in oxygen concentrations in the cell microenvironment may ultimately lead to new engineering tools to direct cellular behavior as well as improved clinical therapeutics for a variety of applications. Unfortunately, most studies in three-dimensional constructs have relied on probe measurements of one area of the sample or mathematical predictions of oxygen diffusion and consumption. Although Clark-type microelectrodes with tip dimensions of up to a few micrometers have been developed [10], they still remain unsuited for measurements of oxygen concentration in laboratory-scale tissue engineering systems because they consume oxygen during operation. Hence, studies in this area have been hindered by the lack of experimental tools to quantitatively and non-invasively measure oxygen concentration in three-dimensional systems.

Here, we present the development and characterization of fluorescent oxygen-sensing microparticles that can be suspended in any transparent biomaterial scaffold used in cell culture and tissue engineering. By monitoring fluorescence quenching of the sensing microparticles with a fluorescence microscope, ratiometric measurements of temporal changes in oxygen concentration can be performed in a rapid, automated, and non-invasive manner without consuming the oxygen available to the cells during culture. Similar sensor technologies have been developed previously, where two recent examples include (1) fluorescent calcium alginate particles surrounded by two polyelectrolyte bilayers for simultaneous measurement of glucose and oxygen concentrations [11], and (2) optical fibers that were modified with a hydrogel matrix containing fluorescent sensor particles for simultaneous measurement of oxygen concentration and $\mathrm{pH}$ [12]. In contrast, we focused on a non-cytotoxic micron-scale particle design to allow for application in three-dimensional investigations of the cell microenvironment. This unique combination of biocompatibility, size, sensitivity to oxygen, and ratiometric response gives these microparticles great potential as tools for quantitatively mapping the fluctuations in oxygen concentration that impact biological responses in cell and tissue engineering studies.

\section{Materials and methods}

All reagents were purchased from Fisher Scientific, Sigma-Aldrich, or otherwise noted.

\subsection{Synthesis of fluorescent oxygen-sensing microparticles}

A suspension of $2 \mathrm{~g}$ of silica gel (Davisil 710, 9.5-11 $\mu$ m-diameter) and $40 \mathrm{~mL}$ of $0.01 \mathrm{~N} \mathrm{NaOH}$ was stirred for $30 \mathrm{~min}$. Next, $10 \mathrm{~mL}$ of a $0.5 \mathrm{~m}$ м solution (in ethanol) of the oxygen-sensitive 
luminophore tris (4,7-diphenyl-1,10-phenanthroline) ruthenium (II) dichloride, or $\mathrm{Ru}$ $\left(\mathrm{Ph}_{2} \mathrm{phen}_{3}\right) \mathrm{Cl}_{2}$ (GFS Chemicals, Powell, $\mathrm{OH}$ ), and $10 \mathrm{~mL}$ of a $0.5 \mathrm{~m}$ solution (also in ethanol) of the oxygen-insensitive fluorophore Nile blue chloride, were added simultaneously. The mixture, deep green in color, was stirred for another $30 \mathrm{~min}$. Next, the solution was centrifuged for $20 \mathrm{~min}$ at $1900 \mathrm{~g}$ and the supernatant was removed. The remaining particles were washed and centrifuged three times in $30 \mathrm{~mL}$ of deionized water and once in $30 \mathrm{~mL}$ of ethanol. After removal of the supernatant, the particles were dried at $70{ }^{\circ} \mathrm{C}$ overnight.

A solution of $0.2 \mathrm{~g}$ of the dry luminophore-bound silica gel particles, $700 \mu \mathrm{L}$ of hexane, $1 \mathrm{~g}$ of poly(dimethylsiloxane) (PDMS) pre-polymer, and $0.1 \mathrm{~g}$ of curing agent (Sylgard 184 elastomer kit; Dow Corning, Midland, MI) was vortexed for $1 \mathrm{~min}$. A $2 \mathrm{w} / \mathrm{v} \%$ solution of sodium dodecyl sulfate (SDS) in water $(300 \mathrm{~mL})$ was heated in a hot-plate to $70^{\circ} \mathrm{C}$ and magnetically stirred at $1200 \mathrm{rpm}$ using a $3.81-\mathrm{cm}(1.5 \mathrm{inch})$ stir bar. The silica gel/PDMS solution was poured into the SDS solution and the resulting oil-in-water emulsion was heated and stirred for 7-8 h. Next, the emulsion was strained twice; first through a $0.5-\mathrm{mm}$ sieve and then through a $25-\mu \mathrm{m}$ sieve. The particles collected by the $25-\mu \mathrm{m}$ sieve were washed and centrifuged three times in $30 \mathrm{~mL}$ of a $0.2 \mathrm{w} / \mathrm{v} \%$ bovine serum albumin (BSA) in phosphate-buffered saline (PBS) solution with $0.1 \%$ methyl-4-hydroxybenzoate (methylparaben) for $20 \mathrm{~min}$ at $1900 \mathrm{~g}$. After the final washing step, the particles were re-suspended to a density of $\sim 50$ particles $/ \mu \mathrm{L}$ in $0.2 \%$ BSA in PBS and stored in the dark at $4{ }^{\circ} \mathrm{C}$.

\subsection{Measurements of microparticle size distribution}

Microparticle size distribution was measured using a Multisizer 3 Coulter counter (BeckmanCoulter, Fullerton, California). Two batches of microparticles were combined and tested in three trials.

\subsection{Cell culture and microparticle cytotoxicity}

Microparticle cytotoxicity was assayed by comparing cell density during culture in the presence of the microparticles to that of the positive control (no particles). Mouse balb/3T3 fibroblasts (ATCC, Manassas, VA) were maintained in high glucose DMEM (Hyclone Laboratories, Logan, Utah) supplemented with 10\% fetal bovine serum (FBS; Invitrogen, Carlsbad, CA), 40 $\mathrm{m}_{\mathrm{ML}}$-glutamine (ATCC, Manassas, VA), and $50 \mu \mathrm{g} / \mathrm{mL}$ of penicillin/streptomycin (MP Biomedicals, Solon, $\mathrm{OH}$ ). Fibroblasts were cultured in 24-well plates (Becton Dickinson, Franklin Lakes, NJ) at a density of 50,000 cells/well $\left(25,000 \mathrm{cells} / \mathrm{cm}^{2}\right)$ for 24 and $48 \mathrm{~h}$ in the presence of 2500 or 5000 particles, which correspond to particle:cell ratios of 1:20 and 1:10, respectively. As a positive control, cells were also cultured under the same conditions without microparticles. The cells were fixed in $4 \%$ formaldehyde in PBS for 20 min. After fixation, cells were washed with PBS to remove the microparticles. Cell nuclei were stained with 300 $\mathrm{n}_{\mathrm{M}}$ 4,6-diamino-2-phenylindole (DAPI) in PBS for $10 \mathrm{~min}$. After mounting with fluoromount$\mathrm{G}$ (Southern Biotech, Birmingham, AL), fluorescence images of adherent cells were captured with an Olympus IX-81 (Olympus, Center Valley, PA) microscope. Image analysis was performed using NIH-ImageJ freeware.

\subsection{Preparation of microparticle-embedded hydrogels}

The poly(ethylene glycol) dimethacrylate (PEG-DM) was synthesized from 8000 Da poly (ethylene glycol). $10 \mathrm{~g}$ of PEG were dried of adsorbed water using azeotropic distillation in toluene ( $4 \mathrm{~mL} / \mathrm{g}$ PEG), concentrated, and then dried overnight under vacuum. Under an argon purge, dry pyridine ( $3 \mathrm{~mL} / \mathrm{g}$ PEG) was added to dissolve the PEG. This solution was cooled to $0^{\circ} \mathrm{C}$ in an ice bath. Methacrylic anhydride (4 molar excess to the moles of PEG) was added slowly with a syringe to the PEG/pyridine solution still in the ice bath. The new solution was mixed in an ice bath for $10 \mathrm{~min}$ and then allowed to react for $48 \mathrm{~h}$ in the dark at room temperature. Working in minimal light, the reaction was diluted $10-15 \times$ with dichloromethane 
and washed twice with $1{ }_{\mathrm{m}} \mathrm{HCl}$ to neutralize the reaction byproducts. The dichloromethane/ PEG-DM solution was dried with sodium sulfate, filtered, concentrated to $50 \mathrm{~mL}$, precipitated in ice-cold diethyl ether, filtered, and dried overnight under vacuum in the dark at room temperature. The PEG-DM product was stored with desiccant at $-20^{\circ} \mathrm{C}$.

Percent methacrylation of PEG-DM was determined by ${ }^{1} \mathrm{H}-\mathrm{NMR}$ (JEOL ECX $400 \mathrm{MHz}$ spectrometer) in deuterated dichloromethane: 1.9 (methacrylate $\mathrm{CH}_{3}$ ), 3.4-3.6 (PEG), 4.2-4.3 (methacrylate $\mathrm{CH}_{2}$ ), 5.5 and 6.0 (methacrylate $\mathrm{CH}$ on PEG-DM), 5.65 and 6.2 (unreacted methacrylic acid $\mathrm{CH}$ ). Peak integration indicated that the degree of methacrylate substitution was $70 \%$ relative to the PEG terminal hydroxyl groups.

A clean glass slide was submerged in a solution of $200 \mathrm{~mL}$ of ethanol, $1 \mathrm{~mL}$ of 3(trimethylsilyl)-propyl methacrylate, and $6 \mathrm{~mL}$ of $10 \%$ acetic acid for $3 \mathrm{~min}$. The glass slide was then rinsed thoroughly with ethanol and air dried. Next, the glass slide was fitted with a casting mold consisting of a 0.5 -mm thick silicone gasket (McMaster-Carr, Robbinsville, $\mathrm{NJ}$ ) coated with vacuum grease. The PEG-DM was crosslinked and covalently bound to the methacrylate-modified glass slide in a free-radical crosslinking reaction: first, $0.015 \mathrm{~g}$ of PEGDM and $1.5 \mu \mathrm{L}$ of $\mathrm{N}, \mathrm{N}, \mathrm{N}^{\prime}, \mathrm{N}^{\prime}$-tetra-methyl-ethylene-diamine (TEMED) were added to $150 \mu \mathrm{L}$ of the $0.2 \mathrm{w} / \mathrm{v} \% \mathrm{BSA} / \mathrm{PBS}$ solution containing $54 \pm 10$ particles $/ \mu \mathrm{L}$ (measured value). Second, $7.5 \mu \mathrm{L}$ of $20 \mathrm{w} / \mathrm{v} \%$ ammonium persulfate (APS) in PBS solution were added to the PEG-DM monomer solution. Finally, the solutions were vortexed, immediately transferred to the glass slide mold, and allowed to react overnight. The construct was stored in water at $4{ }^{\circ} \mathrm{C}$ until use. Average gel thickness after crosslinking was $250 \mu \mathrm{m}$.

Confocal laser microscopy was also employed to image the sensing microparticles inside the PEG-DM hydrogel. Images were taken with a Leica TCS SP5 confocal microscope (Leica Microsystems, Bannockburn, IL) in 5- $\mu \mathrm{m}$ steps from the top to the bottom of the samples. Stacks were reconstructed to create a 3D image of the sensing microparticles throughout the PEG-DM hydrogel using the computer software Volocity (Improvision, Waltham, MA).

\subsection{Parallel-plate flow chamber system}

The microparticle-embedded PEG-DM hydrogels were placed into a parallel-plate flow chamber and applied to calibrate microparticle fluorescence to dissolved oxygen content and determine sensor reversibility. The flow chamber (adapted from Munn et al. [13]), consisted of three sections that were held together by vacuum: (1) a polycarbonate block that served as the top section of the flow area, (2) a 1-mm thick silicone gasket (McMaster-Carr), which determined the total height of the flow area, and (3) a glass slide that served as the bottom of the flow chamber and held the hydrogel sample containing the sensing microparticles. The flow chamber was mounted on the stage of the fluorescence microscope and was connected in circuit form to a 500-mL water reservoir and a peristaltic pump via 1.6-mm ID/4.8-mm OD Tygon tubing (Cole-Parmer, Vernon Hills, IL). Water flow rate through flow chamber system was set at $5 \mathrm{~mL} / \mathrm{min}$. The water reservoir housed a traditional dissolved oxygen meter (VWR, West Chester, PA) and was also connected to two rotameters (Cole-Parmer, Vernon Hills, IL) that were used to control incoming gas flow from a nitrogen tank and a small air pump to the reservoir.

\subsection{Microparticle calibration}

To obtain microparticle fluorescence intensity data as a function of dissolved oxygen content, nitrogen and air were combined to achieve step-wise increments in dissolved oxygen concentration in the flow chamber water reservoir. The reservoir was initially sparged with nitrogen to remove dissolved oxygen from the water. Gas mixture percentages used were 100/0, $94 / 6,85 / 15,70 / 30,60 / 40,40 / 60,30 / 70$, and 0/100 (vol.\% nitrogen)/(vol.\% air). Each step 
change in gas mixture was made after the dissolved oxygen meter indicated that the dissolved oxygen in the reservoir had stabilized. The time elapsed between each step change was 10 min. Oxygen readings from the dissolved oxygen meter and fluorescent images of the sensing microparticles were recorded every $30 \mathrm{~s}$. Each image was captured using a QDOT-605 filter set (Olympus, Center Valley, $\mathrm{PA}$ ) for $\mathrm{Ru}\left(\mathrm{Ph}_{2} \mathrm{phen}_{3}\right) \mathrm{Cl}_{2}$ and a Cy5 filter set (Olympus) for Nile blue chloride. Excitation and emission wavelengths for $\mathrm{Ru}\left(\mathrm{Ph}_{2} \mathrm{phen}_{3}\right) \mathrm{Cl}_{2}$ are 470 and $610 \mathrm{~nm}$, respectively, and Nile blue chloride are 636 and $656 \mathrm{~nm}$, respectively. Exposure times for both $\mathrm{Ru}\left(\mathrm{Ph}_{2}\right.$ phen $\left._{3}\right) \mathrm{Cl}_{2}$ and Nile blue chloride were $100 \mathrm{~ms}$.

Image analysis was conducted using NIH-ImageJ freeware. Using line profiles (or line scans) across the width of a particular image (at $4 \times$ magnification, image area was $2.218 \times 1.651 \mathrm{~mm}$ ), average values of microparticle fluorescence intensity across the surface of the hydrogel were obtained. Each image was segmented with a series of line profiles from which an average value of intensity was determined. Images displaying $\mathrm{Ru}\left(\mathrm{Ph}_{2} \mathrm{phen}_{3}\right) \mathrm{Cl}_{2}$ and Nile blue chloride fluorescence were analyzed separately. Therefore, non-fluorescent particles were omitted from the analysis. Although the image analysis performed for the calibration and reversibility experiments was a global measurement of fluorescence intensity across the image area, the image analysis procedure may also be adapted to measure the intensity of individual particles. Additionally, the analysis may be optimized for application-specific needs as well as the selected concentration and distribution of microparticles in the sample.

To calibrate microparticle fluorescence to dissolved oxygen content, two models were explored. First, we adapted a conventional Stern-Volmer model that describes the relationship between fluorescence intensity of the $\mathrm{Ru}\left(\mathrm{Ph}_{2} \mathrm{phen}_{3}\right) \mathrm{Cl}_{2}$ luminophore and oxygen concentration and normalizes the fluorescence intensity of $\mathrm{Ru}\left(\mathrm{Ph}_{2} \mathrm{phen}_{3}\right) \mathrm{Cl}_{2}$ with the Nile blue chloride reference fluorophore:

$$
\frac{I_{\mathrm{R}, 0}}{I_{\mathrm{R}}}-1=K_{\mathrm{SV}}\left[\mathrm{O}_{2}\right]
$$

where $I_{\mathrm{R}, 0}$ and $I_{\mathrm{R}}$ are the ratios of the fluorescence intensities of $\mathrm{Ru}\left(\mathrm{Ph}_{2} \mathrm{phen}_{3}\right) \mathrm{Cl}_{2}$ and Nile blue chloride in the absence and presence of oxygen respectively, $K_{\mathrm{SV}}$ is the Stern-Volmer quenching constant, and $\left[\mathrm{O}_{2}\right]$ is the concentration of oxygen. Second, we also applied a twosite Stern-Volmer model:

$$
\frac{I_{\mathrm{R}, 0}}{I_{\mathrm{R}}}=\left(\frac{f_{1}}{1+K_{\mathrm{SV}, 1}\left[\mathrm{O}_{2}\right]}+\frac{f_{2}}{1+K_{\mathrm{SV}, 2}\left[\mathrm{O}_{2}\right]}\right)^{-1}
$$

This model, proposed by Carraway et al. [14], states that the oxygen-sensitive luminophore is distributed mainly in two populations through the matrix of a polymer, a quenched population and an unquenched population (with fractions $f_{1}$ and $f_{2}$ respectively), each with its own quenching constant $\left(K_{\mathrm{SV}, 1}\right.$ and $\left.K_{\mathrm{SV}, 2}\right)$. The quenching constant for the unquenched population $\left(K_{\mathrm{SV}, 2}\right)$ is very small and is generally assumed to be negligible. Thus, with this assumption (and the fact that $f_{2}=1-f_{1}$ ), Eq. (2) can then be rearranged to:

$$
\frac{I_{\mathrm{R}, 0}-I_{\mathrm{R}}}{I_{\mathrm{R}, 0}\left(f_{1}-1\right)+I_{\mathrm{R}}}=K_{\mathrm{SV}}\left[\mathrm{O}_{2}\right]
$$


resulting in a simpler two parameter fit (i.e., $f_{1}$ and $K_{\mathrm{SV}}$ ).

\subsection{Microparticle reversibility}

Characterization of microparticle reversibility was carried out in a similar fashion as for the calibration. However, only changes from $100 \%$ nitrogen to $100 \%$ air were employed. As with the microparticle calibration, the changes in dissolved oxygen concentration were made after measured dissolved oxygen inside the flow chamber water reservoir was observed to remain stable. The time elapsed between each change was $12.5 \mathrm{~min}$. Oxygen readings from the dissolved oxygen meter and fluorescent images of the sensing microparticles were recorded every $30 \mathrm{~s}$. System equilibration time, which accounts for time required for oxygen saturation in the water reservoir as well as the sensor response time, was evaluated as the time elapsed to obtain $90 \%$ of the stabilized sensor response after a change from $100 \%$ nitrogen to $100 \%$ air was effected.

\subsection{Statistical analysis}

Statistical analysis for microparticle cytotoxicity and reversibility experiments was performed using ANOVA with a cutoff of $p<0.05$. Statistical analysis for system equilibration times as measured by the microparticles and traditional dissolved oxygen meter was performed using a Student's $t$-test with a cutoff of $p<0.05$.

\section{Results}

\subsection{Microparticle synthesis and size distribution}

Synthesis of the fluorescent oxygen-sensing microparticles consisted of two steps: (1) immobilization of the oxygen-sensitive luminophore $\mathrm{Ru}\left(\mathrm{Ph}_{2} \mathrm{phen}_{3}\right) \mathrm{Cl}_{2}$ and the oxygeninsensitive fluorophore Nile blue chloride to silica gel and (2) the encapsulation of the luminophore-bound silica gel with PDMS via an oil-in-water emulsion. Fluorescent images were captured with a fluorescence microscope after curing of the PDMS. Subsequently, images of the sensing microparticles inside the PEG-DM hydrogel samples were also taken with a confocal microscope. Imaging results are shown in Fig. 1. Fig. 1a shows a fluorescence image of the sensing microparticles, where the red fluorescence marks the $\mathrm{Ru}\left(\mathrm{Ph}_{2} \mathrm{phen}_{3}\right) \mathrm{Cl}_{2}$ luminophore and the blue pseudo-color marks the Nile blue chloride. Fig. 1b shows the same fluorescent image with a phase contrast image overlaid. Fig. 1c displays a 3D reconstruction of the sensing microparticles suspended through the matrix of the hydrogel sample. Confocal z-stacks were also converted to movies that depict the 3D distribution and resolution of the microparticles. (Supplemental Data).

Microparticle size distribution was measured for two combined batches of particles. As shown in Fig. 2, the emulsion process yields fluorescent microparticles wherein $70 \%$ of their size distribution falls in the $5-40 \mu \mathrm{m}$ range.

\subsection{Microparticle cytotoxicity}

To assay microparticle toxicity, balb/3T3 fibroblasts were cultured for up to $48 \mathrm{~h}$ in the presence of the oxygen-sensing microparticles. Cell densities in treated and untreated (positive control) samples were based on counts of the DAPI-stained cell nuclei of adherent cells. Fig. 3 shows cell densities at 24 and $48 \mathrm{~h}$ for cells cultured at particle:cell ratios of 1:10 and 1:20 (the particle:cell ratios were selected based on preliminary studies in two- and three-dimensional systems to probe oxygen content in the local cellular microenvironment). Statistical analysis performed using ANOVA $(p<0.05)$ demonstrated that the cell densities of the treated and untreated wells were not statistically different, affirming that the microparticles were not cytotoxic to the cells under standard cell culture conditions for up to $48 \mathrm{~h}$. 


\subsection{Microparticle calibration}

The calibration of microparticle response when suspended in a three-dimensional hydrogel was performed in a flow chamber such that the oxygen content of the aqueous environment of the hydrogel could be systematically controlled. Nitrogen and air were combined and sparged into the water reservoir to achieve stepwise increments in the aqueous dissolved oxygen concentration. Oxygen concentration inside the water reservoir was monitored with a traditional dissolved oxygen meter. Change in microparticle fluorescence intensity to the changes in oxygen concentration in the water was monitored by capturing fluorescent images of the sensing microparticles with the fluorescence microscope.

To correlate fluorescence intensities to dissolved oxygen content, we explored two models to fit data for the sensing microparticles in the range of $0-160 \mathrm{~mm} \mathrm{Hg}$, or $0-100 \%$ oxygen saturation of the water. Fig. 4a shows the conventional Stern-Volmer calibration (based on Eq. (1)). Regression results were 0.017 for the Stern-Volmer quenching constant $\left(K_{\mathrm{SV}}\right.$, $\mathrm{mmHg}^{-1}$ ) and $R^{2}=0.947$. Fig. $4 \mathrm{~b}$ shows the calibration of the sensing microparticles using the two-site Stern-Volmer equation (based on Eq. (3)). Regression results for the two-site Stern-Volmer model were $0.023 \mathrm{~mm} \mathrm{Hg}^{-1}$ for $K_{\mathrm{SV}}$ and $R^{2}=0.986$. The value obtained for the quenched fluorophore fraction $\left(f_{1}\right)$ was $0.90 \pm 0.02$.

Fig. 4 demonstrates that the sensing microparticles are capable of measuring dissolved oxygen concentrations (partial pressures) in ranges relevant to tissue culture studies; mainly, $<20 \mathrm{mmHg}$ (hypoxic conditions), 30-90 mm Hg (normoxic conditions), and 100-150 mm Hg (hyperoxic conditions).

\subsection{Microparticle reversibility}

To test microparticle reversibility, nitrogen and air were alternated during the course of the experiment. As with calibration, oxygen concentration inside the water reservoir was monitored using the dissolved oxygen meter. Changes in microparticle fluorescence were monitored by capturing images with the fluorescence microscope. These two operations were also carried out simultaneously. Gas content was switched only after the reading from the dissolved oxygen meter had stabilized. Fig. 5a shows microparticle reversibility compared to that of the traditional dissolved oxygen meter. Both the dissolved oxygen meter and the sensing microparticles demonstrated good recovery while the environment was alternated between oxygenated and deoxygenated water. No evidence of hysteresis was observed. Fig. 5b shows the minimum and maximum response (for 100\% air and 100\% nitrogen respectively) of the oxygen-sensing microparticles. Statistical analysis performed using ANOVA $(p<0.05)$ demonstrated that there is no difference within sets of intensity minima and maxima.

System equilibration times as measured by the dissolved oxygen meter and sensing microparticles were (mean \pm standard error) $5.2 \pm 1.0$ and $5.6 \pm 0.4 \mathrm{~min}$, respectively. Student's $t$-test $(p<0.05)$, demonstrated that the two calculated values of equilibration time were not different.

\section{Discussion}

To design the microparticle sensors, $\mathrm{Ru}\left(\mathrm{Ph}_{2} \mathrm{phen}_{3}\right) \mathrm{Cl}_{2}$ was chosen because of its high quantum yield, high oxygen quenching, and thermal stability at physiological temperature $[15,16]$. Synthesis of the sensing microparticles is a simple and inexpensive two-step process. The first step consisted of immobilization of $\mathrm{Ru}\left(\mathrm{Ph}_{2} \mathrm{phen}_{3}\right) \mathrm{Cl}_{2}$ and Nile blue chloride, to silica gel particles $9.5-11 \mu \mathrm{m}$ in diameter. The reference fluorophore, Nile blue chloride, allows ratiometric (or normalized) measurements that correct for the typical drawbacks of fluorescence intensity measurements such as bleaching, heterogeneous fluorophore 
concentration, fluctuations in excitation light intensity, and camera sensitivity. The immobilization process was adapted from John et al. [17], who immobilized $\mathrm{Ru}\left(\mathrm{Ph}_{2} \mathrm{phen}_{3}\right)$ $\mathrm{Cl}_{2}$ and the reference fluorophore safranin onto silica gel. In contrast, we used Nile blue chloride as our reference fluorophore due to the fact that safranin's emission spectrum overlaps with that of $\mathrm{Ru}\left(\mathrm{Ph}_{2} \mathrm{phen}_{3}\right) \mathrm{Cl}_{2}$. The differing emission spectra between the $\mathrm{Ru}\left(\mathrm{Ph}_{2} \mathrm{phen}_{3}\right) \mathrm{Cl}_{2}$ and the Nile blue chloride allowed us to identify and independently measure their fluorescence inside the microparticles with the fluorescence microscope. Conversely, the absorption spectrum of Nile blue chloride was found to overlap with the emission spectrum of Ru $\left(\mathrm{Ph}_{2} \mathrm{phen}_{3}\right) \mathrm{Cl}_{2}$, which might lead to fluorescence resonance energy transfer (FRET) between the two fluorophores. Thus, negligible FRET was a key criterion in the development of the reported microparticle synthesis procedure. The absence of FRET was confirmed in gas phase experiments using PDMS films containing silica gel bound with $\mathrm{Ru}\left(\mathrm{Ph}_{2} \mathrm{phen}_{3}\right) \mathrm{Cl}_{2}$ and Nile blue chloride (data not shown).

The second microparticle synthesis step consisted of the encapsulation of the modified silica gel within PDMS. PDMS is optically transparent, hydrophobic, non-cytotoxic, and highly permeable to gases such as oxygen, nitrogen, and carbon dioxide [18]. For instance, the diffusivity of oxygen in PDMS is approximately twice that of oxygen in water $\left(4.1 \times 10^{-5}\right.$ $\mathrm{cm}^{2} / \mathrm{s}$ and $2.1 \times 10^{-5} \mathrm{~cm}^{2} / \mathrm{s}$ for PDMS and water respectively at room temperature) [19]. Therefore, by encapsulating the $\mathrm{Ru}\left(\mathrm{Ph}_{2} \mathrm{phen}_{3}\right) \mathrm{Cl}_{2^{-}}$and Nile blue chloride-modified silica gel within PDMS, we ensure relatively unrestricted oxygen transport from the culture medium to the luminophore. Moreover, preliminary cell culture studies indicated that cell viability was not adversely affected by the presence of the microparticles in the cell microenvironment (Fig. 3 ), which suggests that encapsulation in PDMS also provides a protective barrier to inhibit luminophore leaching into the cell culture environment.

The emulsion process yielded microparticles that were predominantly in the 5-40 $\mu \mathrm{m}$ range (Fig. 2). This size range is optimal for the sensing microparticles, as it is on the size range of cells, yet large enough to prevent endocytosis. Furthermore, this small size range minimizes diffusion limitations of oxygen from the medium to the luminophore and significantly reduces sensor response time [20,21]. Due to the range in microparticle size, heterogeneity in their distribution through the hydrogel volume was expected. We compensated for this effect by using high particle concentrations $(54 \pm 10$ particles $/ \mu \mathrm{L})$ in the PEG-DM hydrogels.

Microparticle calibration inside a three-dimensional hydrogel and characterization of the microparticle reversibility were carried out using a parallel-plate flow chamber mounted on a fluorescence microscope stage. In addition, to demonstrate the functionality of the sensing microparticles, their reversibility was compared to that of a traditional dissolved oxygen meter. The flow chamber system allowed for systematic control of the dissolved oxygen in the microparticle environment as well as simultaneous measurements of oxygen concentration and changes in microparticle fluorescence intensity. The oxygen concentration of the inlet solution was monitored with a dissolved oxygen meter while the response of the microparticle sensors was monitored via fluorescence images captured at the midpoint of the flow chamber length.

Retaining the reversibility of the $\mathrm{Ru}\left(\mathrm{Ph}_{2} \mathrm{phen}_{3}\right) \mathrm{Cl}_{2}$ luminophore after the immobilization and encapsulation steps is important for sensor operation in environments with fluctuating oxygen concentration. As shown in Fig. 5a and b, both the dissolved oxygen meter and the microparticle sensors demonstrated good recovery. Statistical analysis demonstrated that there is no difference within sets of normalized intensity minima and maxima. No evidence of hysteresis was observed, as both responses were capable of returning to their initial state between cycles. Also, using both the microparticles, as well as the traditional dissolved oxygen probe, we determined the time for system equilibration, which accounts for the time required for oxygen saturation in the water reservoir as well as the sensor response time. The system equilibration 
times are equal, suggesting that the response time of both sensors in the flow-loop apparatus is limited by the rate of oxygen transport through water rather than sensor performance.

Calibration of microparticle fluorescence intensity to dissolved oxygen content (Fig. 4) demonstrates that the microparticle sensors were capable of measuring oxygen partial pressures in ranges relevant to tissue culture studies. It is interesting to note that the conventional SternVolmer model (Eq. (1)), which pertains to luminophore in solution, predicts a linear response of the $\mathrm{Ru}\left(\mathrm{Ph}_{2} \mathrm{phen}_{3}\right) \mathrm{Cl}_{2}$ luminophore to oxygen. However, when the luminophore is embedded in a polymeric support, its response ceases to be linear (Fig. 4a). The demonstrated downward curvature is expected due to heterogeneities in the polymer structure and non-uniform distribution of luminophore molecules throughout a polymer matrix [14,16,22]. Hence, the two-site model of the Stern-Volmer equation (Eq. (3)) was also applied.

As demonstrated by the regression results (Fig. 4b), the two-site Stern-Volmer equation is a better fit for the calibration data than the conventional Stern-Volmer model. The value obtained for the quenched luminophore fraction $\left(f_{1}\right)$ was $0.90 \pm 0.02$. Reported values for $f_{1}$ have ranged from 0.97 for the $\mathrm{Ru}\left(\mathrm{Ph}_{2} \mathrm{phen}_{3}\right) \mathrm{Cl}_{2}$ luminophore in PDMS films [14], to 0.73 in poly(styrene) films [23], and to 0.61 while adsorbed on mesoporous silica particles suspended in selfassembled polymer films [24]. The value obtained from our experiments is closer to that obtained by Carraway et al. [14], which pertains to $\mathrm{Ru}\left(\mathrm{Ph}_{2} \mathrm{phen}_{3}\right) \mathrm{Cl}_{2}$ in PDMS. Our result reflects that approximately $90 \%$ of the available oxygen-sensitive luminophore is quenched under our particular testing conditions. The differences observed in the reported values of $f_{1}$ suggest that this value is mainly dependent on the material contacting the luminophore. For example, the polarity of the environment in which the $\mathrm{Ru}\left(\mathrm{Ph}_{2} \mathrm{phen}_{3}\right) \mathrm{Cl}_{2}$ luminophore is present affects fluorescence quenching by oxygen. Xu et al. [22] demonstrated that when the Ru $\left(\mathrm{Ph}_{2} \mathrm{phen}_{3}\right) \mathrm{Cl}_{2}$ luminophore was immobilized onto a polar carrier, such as silica, and added to a non-polar silicone polymer material, fluorescence quenching by oxygen was enhanced. They speculated that the luminophore was partitioned between two fractions, one present in the polar silica and one preset in the non-polar silicone polymer, resulting in decreased fluorescence quenching by oxygen and a downward curvature observed in the Stern-Volmer plot. This partition was due to the low solubility of the luminophore in the non-polar polymer. Therefore, one possible explanation for the value we have obtained for quenched luminophore fraction $\left(f_{1}\right)$ is that it corresponds to a fraction of luminophore that exists immobilized onto the silica gel $(90 \%)$; where as, the remaining fraction $(10 \%)$ exists, or is in contact with, the PDMS. This partition decreases fluorescence quenching by oxygen of the luminophore and deviates the Stern-Volmer calibration of the sensing microparticles from linearity. These results demonstrate that the silica/PDMS system we have employed was a suitable choice for our design. Moreover, PDMS is non-cytotoxic, transparent, and easy to process; thus, the reported oxygen-sensing microparticle system presents significant advantages in terms of performance as well as ease of manufacturing and application in cell culture studies using a readily adaptable fluorescence microscopy analysis.

Future work will take advantage of the versatility of the sensing microparticles by demonstrating that dynamic correlations between cell function and oxygen concentration in engineered tissues can be achieved in two-dimensional as well as three-dimensional environments.

\section{Conclusions}

Here, we have described the development and characterization of fluorescent oxygen-sensing microparticles. We have demonstrated that the luminophore-bound microparticles can be suspended in a transparent biomaterial and can be used to obtain ratiometric measurements of temporal changes in oxygen concentrations by monitoring fluorescence intensity with a 
fluorescence microscope. Synthesis of the microparticles is a simple two-step process, which yielded microparticles in a size distribution predominantly in the 5-40 $\mu \mathrm{m}$ range. The microparticles were demonstrated to be non-cytotoxic to cells during culture. Sensing microparticle response showed good reversibility and no evidence of hysteresis was observed. Additionally, the measured system equilibration time was comparable to that of a traditional dissolved oxygen meter and calibration demonstrated that the sensors were capable of measuring oxygen partial pressures in ranges relevant to tissue culture studies. While the conventional Stern-Volmer model showed acceptable results for fitting the microparticle calibration data, the two-site Stern-Volmer model resulted in the best fitting results and the calculated fraction of quenched $\mathrm{Ru}\left(\mathrm{Ph}_{2} \mathrm{phen}_{3}\right) \mathrm{Cl}_{2}$ for the sensing microparticles was close to that reported in literature for the same luminophore in PDMS.

\section{Supplementary Material}

Refer to Web version on PubMed Central for supplementary material.

\section{Acknowledgments}

We thank Julia M. Ross for valuable technical discussions and for use of the Coulter counter, Mark R. Marten and Judith K. Pollack for providing the parallel-plate flow chamber, Chere Petty for assistance with confocal microscopy, and Michel Wathier, Robert Reeves, Leonard Lombardo, and Silviya Petrova for assistance with the synthesis of the PEG-DM. This work was supported by the Henry Luce Foundation and UMBC. The Leica TCS SP5 confocal microscope was funded by NSF DBI-0722569.

\section{Appendix}

Figures with essential color discrimination. Parts of Fig. 1 of this article are difficult to interpret in black and white. The full color images can be found in the on-line version, at 10.1016/ j.biomaterials.2009.02.021.

\section{References}

1. Bussink J, Kaanders JH, Strik AM, Vojnovic B, van Der Kogel AJ. Optical sensor-based oxygen tension measurements correspond with hypoxia marker binding in three human tumor xenograft lines. Radiat Res 2000;154(5):547-55. [PubMed: 11025651]

2. Moeller BJ, Richardson RA, Dewhirst MW. Hypoxia and radiotherapy: opportunities for improved outcomes in cancer treatment. Cancer Metastasis Rev 2007;26(2):241-8. [PubMed: 17440683]

3. Dewhirst MW, Cao Y, Moeller B. Cycling hypoxia and free radicals regulate angiogenesis and radiotherapy response. Nat Rev Cancer 2008;8(6):425-37. [PubMed: 18500244]

4. Martin H, Sarsat JP, Lerche-Langrand C, Housset C, Balladur P, Toutain H, et al. Morphological and biochemical integrity of human liver slices in long-term culture: effects of oxygen tension. Cell Biol Toxicol 2002;18(2):73-85. [PubMed: 12046692]

5. Fariss MW. Oxygen toxicity: unique cytoprotective properties of vitamin E succinate in hepatocytes. Free Radic Biol Med 1990;9(4):333-43. [PubMed: 2283088]

6. Martin Y, Vermette P. Bioreactors for tissue mass culture: design, characterization, and recent advances. Biomaterials 2005;26(35):7481-503. [PubMed: 16023202]

7. Radisic M, Malda J, Epping E, Geng W, Langer R, Vunjak-Novakovic G. Oxygen gradients correlate with cell density and cell viability in engineered cardiac tissue. Biotechnol Bioeng 2006;93(2):33243. [PubMed: 16270298]

8. O'Driscoll SW, Fitzsimmons JS, Commisso CN. Role of oxygen tension during cartilage formation by periosteum. J Orthop Res 1997;15(5):682-7. [PubMed: 9420597]

9. Westfall SD, Sachdev S, Das P, Hearne LB, Hannink M, Roberts RM, et al. Identification of oxygensensitive transcriptional programs in human embryonic stem cells. Stem Cells Dev 2008;17(5):86981. [PubMed: 18811242] 
10. Revsbech NP. An oxygen microsensor with a guard cathode. Limnol Oceanogr 1989;34(2):474-8.

11. Brown JQ, Srivastava R, McShane MJ. Encapsulation of glucose oxidase and an oxygen-quenched fluorophore in polyelectrolyte-coated calcium alginate microspheres as optical glucose sensor systems. Biosens Bioelectron 2005;21(1):212-6. [PubMed: 15967372]

12. Vasylevska GS, Borisov SM, Krause C, Wolfbeis OS. Indicator-loaded permeation-selective microbeads for use in fiber optic simultaneous sensing of ph and dissolved oxygen. Chem Mater 2006;18:4609-16.

13. Munn LL, Melder RJ, Jain RK. Analysis of cell flux in the parallel plate flow chamber: implications for cell capture studies. Biophys J 1994;67(2):889-95. [PubMed: 7948702]

14. Carraway ER, DeGraff BA, Bacon JR, Demas JN. Photophysics and photochemistry of oxygen sensors based on luminescent transition-metal complexes. Anal Chem 1991;63:337-42.

15. Kneas KA, Xu W, Demas JN, DeGraff BA. Oxygen sensors based on luminescence quenching: interaction of tris(4,7-diphenyl-1,10-phenanthroline) ruthenium(II) chloride and pyrene with polymer supports. Appl Spectrosc 1997;51(9):1346-51.

16. Bacon JR, Demas JN. Determination of oxygen concentrations by luminescence quenching of a polymer-immobilized transition metal complex. Anal Chem 1987;59:2780-5.

17. John GT, Klimant I, Wittman C, Hienzle E. Integrated optical sensing of dissolved oxygen in microtiter plates: a novel tool for microbial cultivation. Biotechnol Bioeng 2003;81(7):829-36. [PubMed: 12557316]

18. McDonald JC, Duffy DC, Andreson JR, Chiu DT, Wu H, Schueller OJ, et al. Fabrication of microfluidic systems in poly(dimethylsiloxane). Electrophoresis 2000;21(1):27-40. [PubMed: 10634468]

19. Charati SG, Stern SA. Diffusion of gases in silicone polymers: molecular dynamics simulations. Macromolecules 1998;31:5529-35.

20. Klimant I, Ruckruh F, Liebsch G, Stangelmayer A, Wolfbeis OS. Fast response oxygen micro-optodes based on novel soluble ormosil glasses. Mikrochimica Acta 1999;131:35-46.

21. Xu H, Aylott JW, Kopelman R, Miller TJ, Philbert MA. A real-time ratiometric method for the determination of molecular oxygen inside living cells using sol-gel-based spherical optical nanosensors with applications to rat C6 glioma. Anal Chem 2001;73(17):4124-33. [PubMed: 11569801]

22. Xu W, McDonough RC, Langsdorf B, Demas JN, DeGraff BA. Oxygen sensors based on luminescence quenching: interactions of metal complexes with the polymer supports. Anal Chem 1994;66:4133-41. [PubMed: 7847622]

23. Borisov SM, Klimant I. Ultrabright oxygen optodes based on cyclometalated iridium(III) coumarin complexes. Anal Chem 2007;79(19):7501-9. [PubMed: 17718539]

24. Han B, Manners I, Winnik MA. Oxygen sensors based on mesoporous silica particles on layer-bylayer self-assembled films. Chem Mater 2005;17(12):3160-71. 

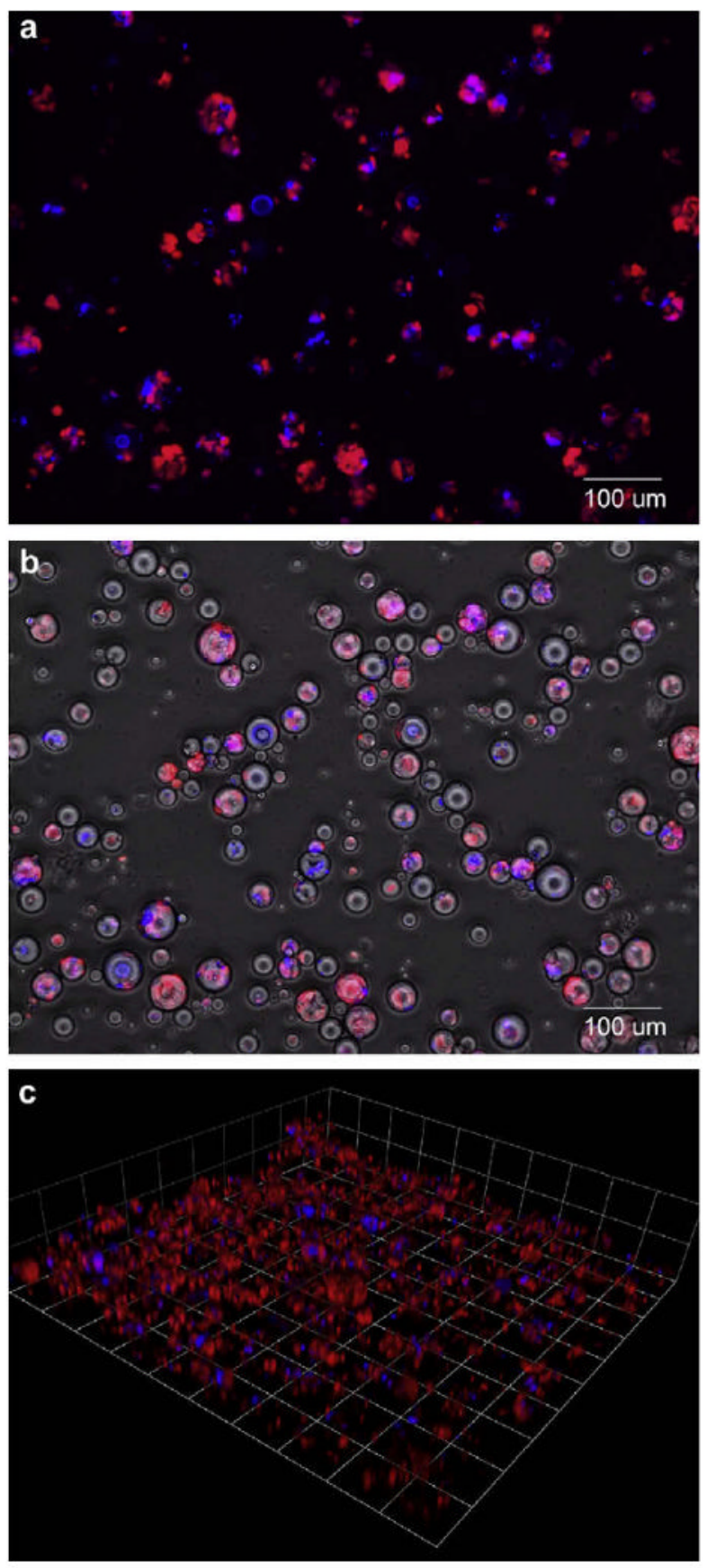

Fig. 1.

Images of oxygen-sensing microparticles captured after curing of PDMS. (a) Red fluorescence marks the oxygen-sensitive luminophore $\mathrm{Ru}\left(\mathrm{Ph}_{2} \mathrm{phen}_{3}\right) \mathrm{Cl}_{2}$ and blue pseudo-color marks the reference fluorophore Nile blue chloride. (b) Fluorescent image of sensing microparticles with phase contrast image overlaid. (c) Confocal image of sensing microparticles distributed throughout matrix of PEG-DM hydrogel sample $(1$ grid unit $=150 \mu \mathrm{m})$. Movies of confocal z-stacks are also available in the Supplemental Data. 


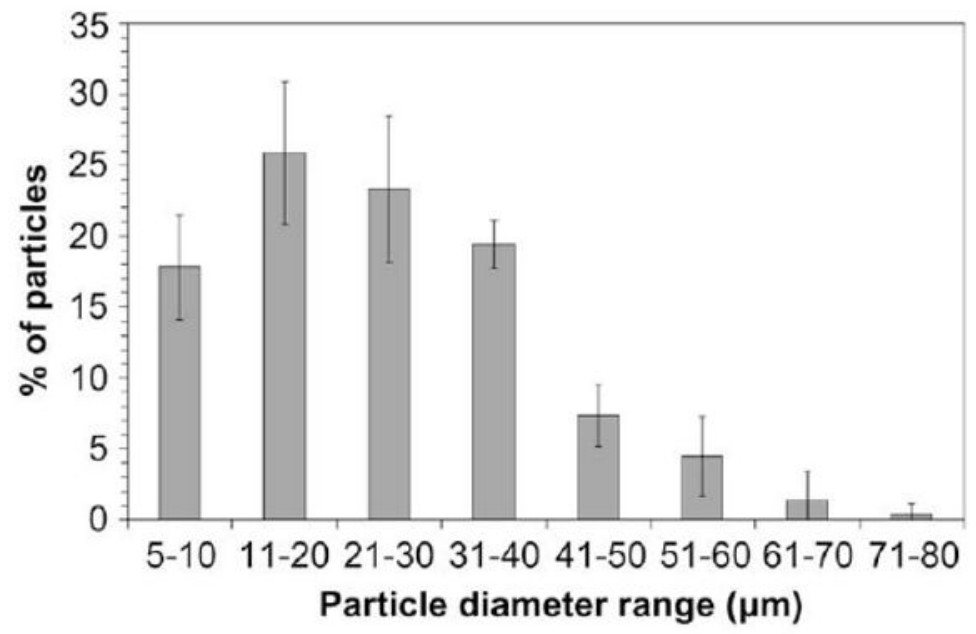

Fig. 2.

Size distribution for oxygen-sensing microparticles as measured with a Coulter counter. Data shown as mean \pm standard error for two batches combined and tested in three trials. 


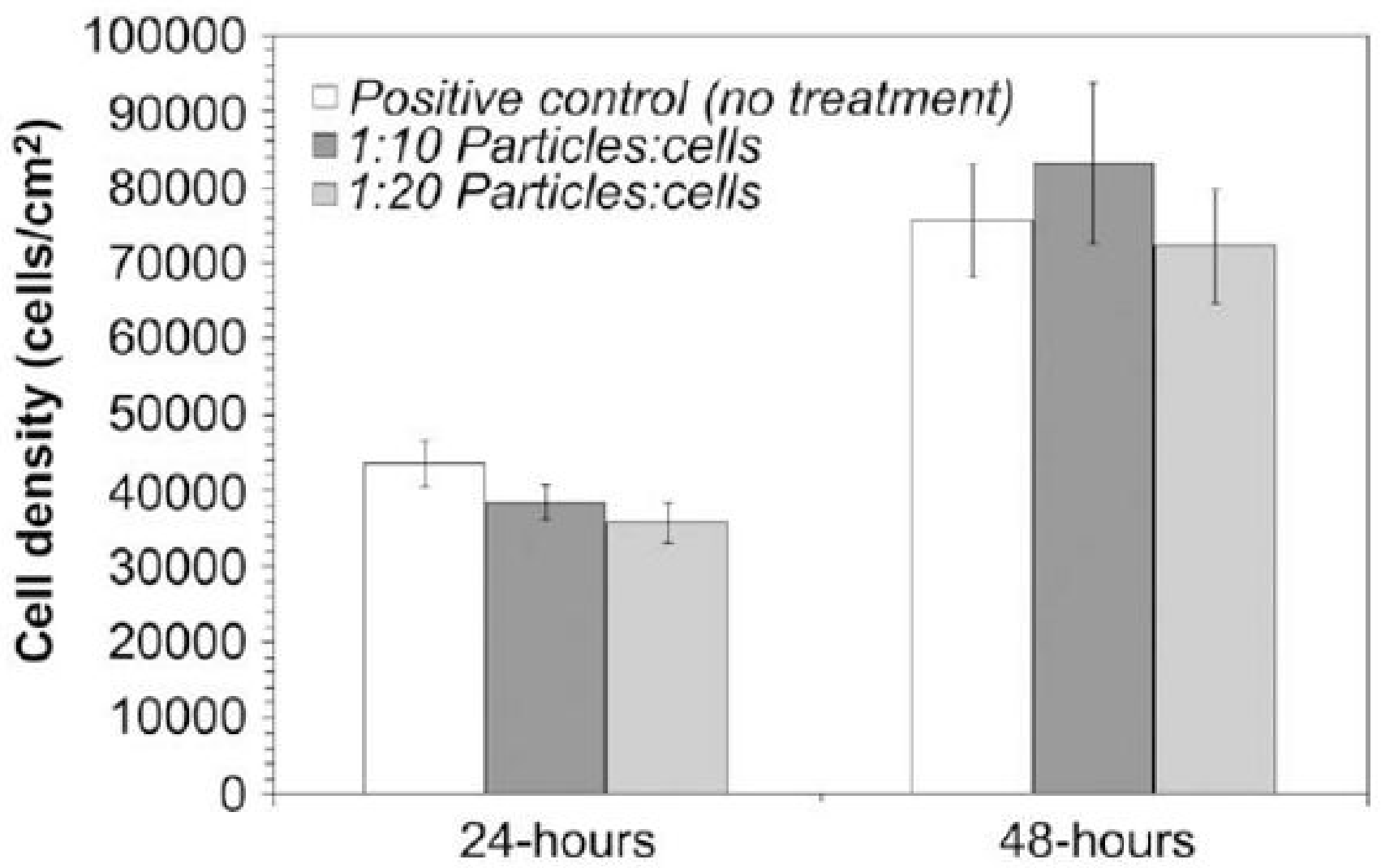

Fig. 3.

Cell densities (in cells $/ \mathrm{cm}^{2}$ ) at 24 and $48 \mathrm{~h}$ for Balb/3T3 mouse fibroblasts cultured in the presence of two oxygen-sensing microparticle densities representative of particle:cell ratios useful for probing cell microenvironment in two- and three-dimensional studies. ANOVA analysis indicated that there is no statistical difference between treatments at each time point, signifying that the microparticles are non-cytotoxic to the cells under standard in vitro conditions. Data shown as mean \pm standard error for six trials. 

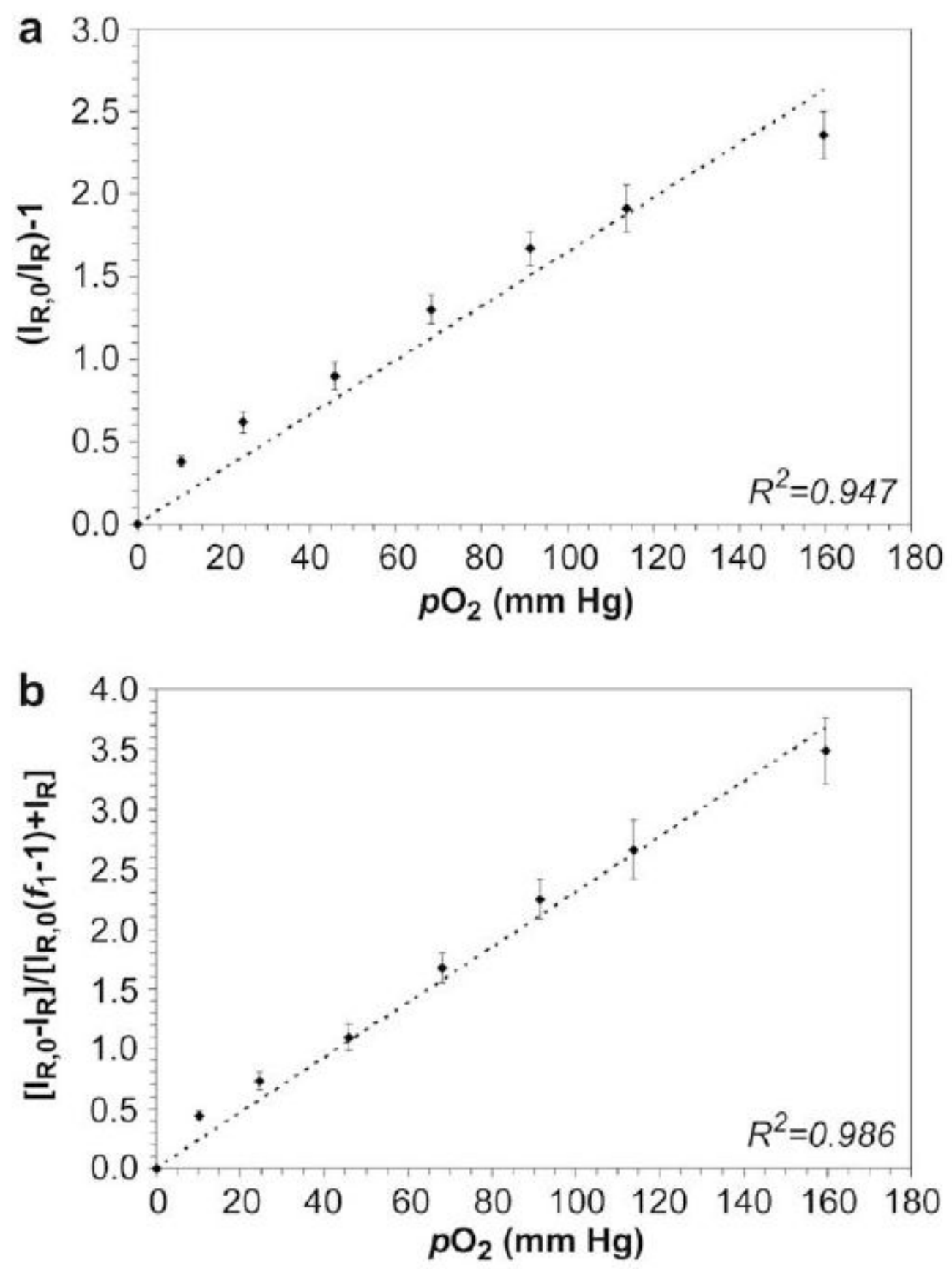

Fig. 4.

Calibration of oxygen-sensing microparticles using the conventional Stern-Volmer model (a; $\left.R^{2}=0.947\right)$ and the two-site Stern-Volmer model $\left(\mathbf{b} ; R^{2}=0.986\right)$. Data points in each plot are shown as mean \pm standard error of normalized intensity data obtained from three calibrations performed on microparticles at the surface of a three-dimensional PEG-DM hydrogel sample. Model fits are represented by dashed lines. 

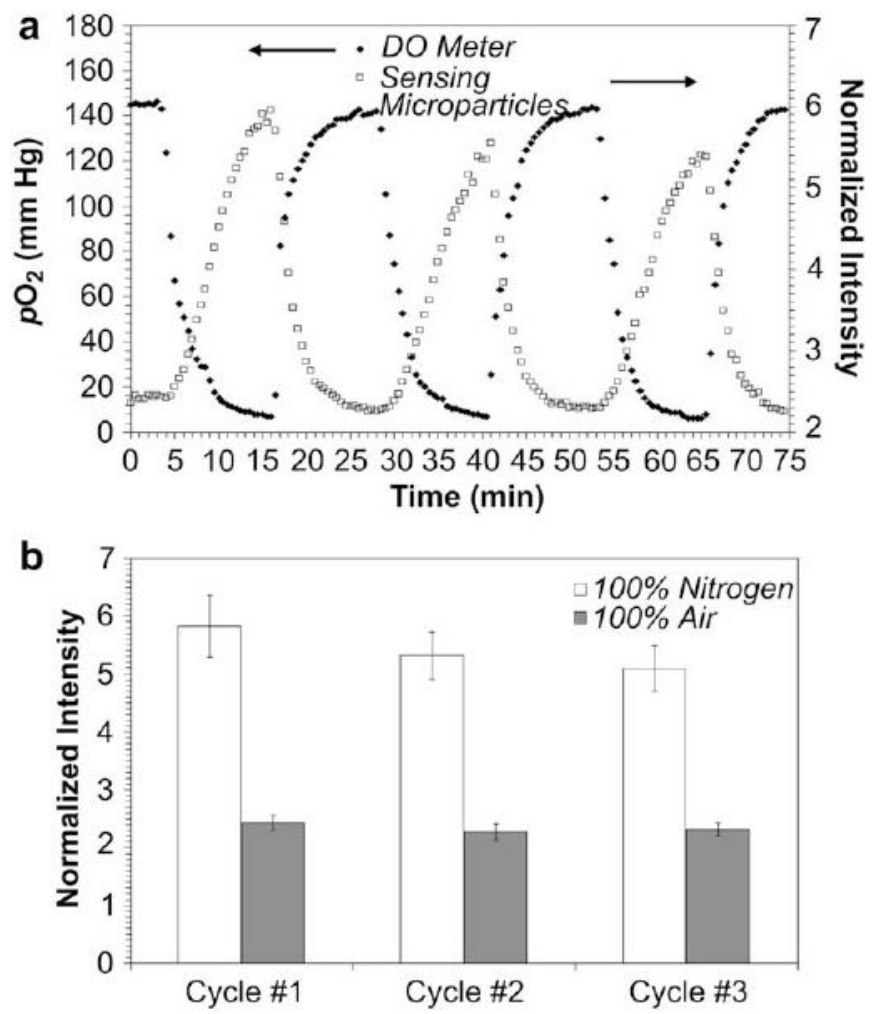

Fig. 5.

(a) Typical reversibility response of oxygen-sensing microparticles compared to that of traditional dissolved oxygen meter. Arrows displayed on plot legend point to respective axes; left axis for dissolved oxygen meter data and right axis for microparticle data. (b) Normalized intensity values of oxygen-sensing microparticles for low and high oxygen content (water reservoir sparge of $100 \%$ nitrogen and $100 \%$ air, respectively) during reversibility experiments. ANOVA analysis indicated that there is no statistical difference between the sets of intensity minima and maxima measured at both conditions. Data shown as mean \pm standard error for three trials. 